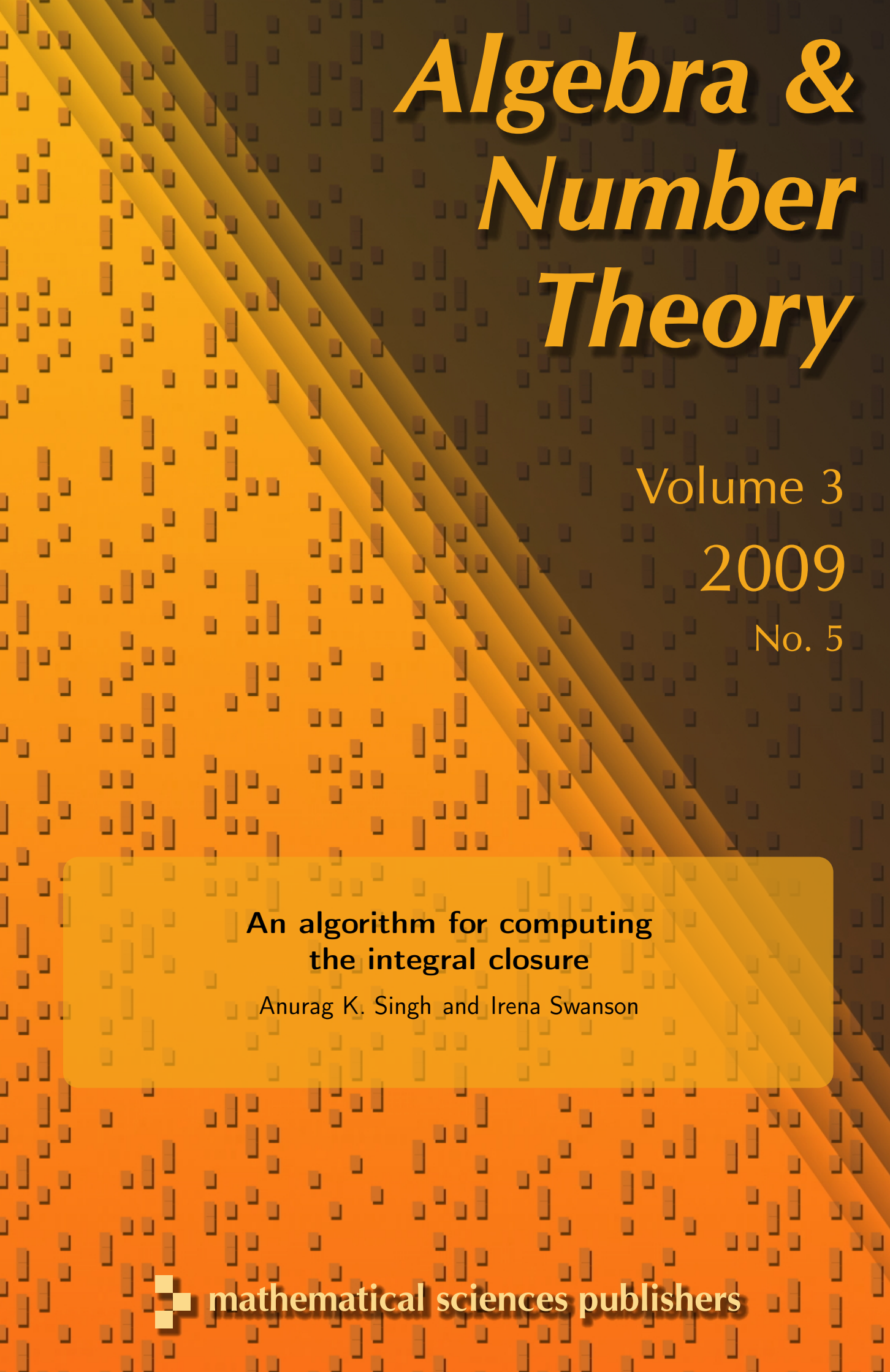




\title{
An algorithm for computing the integral closure
}

\author{
Anurag K. Singh and Irena Swanson
}

We present an algorithm for computing the integral closure of a reduced ring that is finitely generated over a finite field.

Leonard and Pellikaan [2003] devised an algorithm for computing the integral closure of weighted rings that are finitely generated over finite fields. Previous algorithms proceed by building successively larger rings between the original ring and its integral closure [de Jong 1998; Seidenberg 1970; 1975; Stolzenberg 1968; Vasconcelos 1991; 2000]; the Leonard-Pellikaan algorithm instead starts with the first approximation being a finitely generated module that contains the integral closure, and successive steps produce submodules containing the integral closure. The weights in [Leonard and Pellikaan 2003] impose strong restrictions, and play a crucial role in various steps of their algorithm; see Remark 1.7. We present a modification of the Leonard-Pellikaan algorithm that works in much greater generality: it computes the integral closure of a reduced ring that is finitely generated over a finite field.

We discuss an implementation of the algorithm in Macaulay 2, and provide comparisons with de Jong's algorithm [1998].

\section{The algorithm}

Our main result is the following theorem; see Remark 1.5 for an algorithmic construction of an element $D$ as below when $R$ is a domain, and for techniques for dealing with the more general case of reduced rings.

Theorem 1.1. Let $R$ be a reduced ring that is finitely generated over a computable field of characteristic $p>0$. Set $\bar{R}$ to be the integral closure of $R$ in its total ring of fractions. Suppose $D$ is a nonzerodivisor in the conductor ideal of $R$, that is, $D$ is a nonzerodivisor with $D \bar{R} \subseteq R$.

MSC2000: primary 13B22; secondary 13P99, $13 \mathrm{~A} 35$.

Keywords: integral closure, algorithm, prime characteristic.

Singh was supported by NSF grant DMS 0600819. 
(1) Set $V_{0}=\frac{1}{D} R$, and inductively define $V_{e+1}=\left\{f \in V_{e} \mid f^{p} \in V_{e}\right\}$ for $e \geqslant 0$. Then the modules $V_{e}$ are algorithmically constructible.

(2) The descending chain $V_{0} \supseteq V_{1} \supseteq V_{2} \supseteq V_{3} \supseteq \cdots$ stabilizes. If $V_{e}=V_{e+1}$, then $V_{e}$ equals $\bar{R}$.

The prime characteristic enables us to use the Frobenius or $p$ th power map; this is what makes the modules $V_{e}$ algorithmically constructible.

Remark 1.2. For each integer $e \geqslant 0$, the module $D V_{e}$ is an ideal of $R$; we set $U_{e}=D V_{e}$ and use this notation in the proof of Theorem 1.1 as well as in the Macaulay 2 code in the following section. The inductive definition of $V_{e}$ translates to $U_{0}=R$ and $U_{e+1}=\left\{r \in U_{e} \mid r^{p} \in D^{p-1} U_{e}\right\}$ for $e \geqslant 0$.

Proof of Theorem 1.1. (1) By Remark 1.2, it suffices to establish that the ideals $U_{e}$ are algorithmically constructible. This follows inductively since

$$
U_{e+1}=U_{e} \cap \operatorname{ker}\left(R \stackrel{F}{\longrightarrow} R \stackrel{\pi}{\longrightarrow} R / D^{p-1} U_{e}\right) \text { for } e \geqslant 0,
$$

where $F$ is the Frobenius endomorphism of $R$, and $\pi$ the canonical surjection.

(2) By construction, one has $V_{e+1} \subseteq V_{e}$ for each $e$. Moreover, it is a straightforward verification that

$$
V_{e}=\left\{f \in V_{0} \mid f^{p^{i}} \in V_{0} \text { for each } i \leqslant e\right\} .
$$

Suppose $f \in \bar{R}$. Then $f^{p^{i}} \in \bar{R}$ for each $i \geqslant 0$, so $D f^{p^{i}} \in R$. It follows that $f \in V_{e}$ for each $e$.

If $V_{e+1}=V_{e}$ for some positive integer $e$, then it follows from the inductive definition that $V_{e+i}=V_{e}$ for each $i \geqslant 1$.

Let $v_{1}, \ldots, v_{s}: R \longrightarrow \mathbb{Z} \cup\{\infty\}$ be the Rees valuations of the ideal $D R$, that is, $v_{i}$ are valuations such that for each $n \in \mathbb{N}$, the integral closure of the ideal $D^{n} R$ equals $\left\{r \in R \mid v_{i}(r) \geqslant n v_{i}(D)\right.$ for each $\left.i\right\}$. Let $e$ be an integer such that $p^{e}>v_{i}(D)$ for each $i$. Suppose $r / D \in V_{e}$. Then $(r / D)^{p^{e}} \in V_{0}$, so $r^{p^{e}} \in D^{p^{e}-1} R$. It follows that $p^{e} v_{i}(r) \geqslant\left(p^{e}-1\right) v_{i}(D)$ for each $i$, and hence that

$$
v_{i}(r) \geqslant v_{i}(D)-v_{i}(D) / p^{e}>v_{i}(D)-1
$$

for each $i$. Since $v_{i}(r)$ is an integer, it follows that $v_{i}(r) \geqslant v_{i}(D)$ for each $i$, and therefore $r \in \overline{D R}$. But then $r$ belongs to the integral closure of the ideal $D \bar{R}$ in $\bar{R}$. Since principal ideals are integrally closed in $\bar{R}$, it follows that $r \in D \bar{R}$, whence $r / D \in \bar{R}$.

Remark 1.3. If $R$ is an integral domain satisfying the Serre condition $S_{2}$, then each module $V_{e}$ is $S_{2}$ as well:

Proceed by induction on $e$. Without loss of generality, assume $R$ is local. Let $x, y$ be part of a system of parameters for $R$. Suppose $y v \in x V_{e+1}$ for an element 
$v \in V_{e+1}$. Then $y v / x \in V_{e+1}$, that is, $y v / x \in V_{e}$ and $y^{p} v^{p} / x^{p} \in V_{e}$, or equivalently, $y v \in x V_{e}$ and $y^{p} v^{p} \in x^{p} V_{e}$. Since $V_{e}$ is $S_{2}$ by the inductive hypothesis, it follows that $v \in x V_{e}$ and $v^{p} \in x^{p} V_{e}$, hence $v \in x V_{e+1}$.

Remark 1.4. In the notation of Theorem 1.1, suppose $e$ is an integer such that $V_{e}=V_{e+1}$. We claim that the integral closure of a principal ideal $a R$ is

$$
\left\{r \in R \mid D r^{p^{i}} \in a^{p^{i}} R \text { for each } i \leqslant e+1\right\} .
$$

To see this, suppose $r$ is an element of the ideal displayed above. Then $D r^{p}=g a^{p}$ for some $g \in R$. Since

$$
D(r / a)^{p^{i}} \in R \quad \text { for each } i \leqslant e+1,
$$

it follows that

$$
D(g / D)^{p^{i}} \in R \quad \text { for each } i \leqslant e .
$$

But then $g / D \in V_{e}$, which implies that $g / D \in V_{i}$ for each $i$. Hence $D(r / a)^{p^{i}} \in R$ for each $i$, equivalently $r \in \overline{a R}$.

Remark 1.5. Let $R$ be a reduced ring that is finitely generated over a perfect field $K$ of prime characteristic $p$. We describe how to algorithmically obtain a nonzerodivisor $D$ in the conductor ideal of $R$.

Case 1. Suppose $R$ is an integral domain. Consider a presentation of $R$ over $K$, say $R=K\left[x_{1}, \ldots, x_{n}\right] /\left(f_{1}, \ldots, f_{m}\right)$. Set $h=$ height $\left(f_{1}, \ldots, f_{m}\right)$. Then the determinant of each $h \times h$ submatrix of the Jacobian matrix $\left(\partial f_{i} / \partial x_{j}\right)$ multiplies $\bar{R}$ into $R$; this may be concluded from the Lipman-Sathaye Theorem [1981] (also found as Theorem 12.3.10 in [Huneke and Swanson 2006]), as discussed in the following paragraph. At least one such determinant has nonzero image in $R$, and can be chosen as the element $D$ in Theorem 1.1. Other approaches to obtaining an element $D$ are via the proof of [Huneke and Swanson 2006, Theorem 3.1.3], or equivalently, via the results from [Stichtenoth 1993].

Let $J$ be the ideal of $R$ generated by the images of the $h \times h$ submatrices of $\left(\partial f_{i} / \partial x_{j}\right)$. We claim that $J$ is contained in the conductor of $R$. By passing to the algebraic closure, assume $K$ is algebraically closed. After a linear change of coordinates, assume that the $x_{i}$ are in general position, specifically, that for any $n-h$ element subset $\Lambda$ of $\left\{x_{1}, \ldots, x_{n}\right\}$, the extension $K[\Lambda] \subseteq R$ is a finite integral extension, equivalently that $K[\Lambda]$ is a Noether normalization of $R$. By the Lipman-Sathaye Theorem, the relative Jacobian $J_{R / K[\Lambda]}$ is contained in the conductor ideal. The claim now follows since, as $\Lambda$ varies, the relative Jacobian ideals $J_{R / K[\Lambda]}$ generate the ideal $J$.

Even when $R$ is not necessarily an integral domain, the ideal $J$, as defined above, is not contained in any minimal prime of $R$; this follows from the Jacobian criterion, see, for example, [Huneke and Swanson 2006, Theorem 4.4.9]. 
Case 2. In the case where $R$ is a reduced equidimensional ring, one may proceed as above and choose $D$ to be the determinant of an $h \times h$ submatrix of $\left(\partial f_{i} / \partial x_{j}\right)$, and then test to see whether $D$ is a nonzerodivisor. If it turns out that $D$ is a nonzero zerodivisor, set $I_{1}=\left(0:_{R} D\right)$ and $I_{2}=\left(0:_{R} I_{1}\right)$. Then each of $R / I_{1}$ and $R / I_{2}$ is a reduced equidimensional ring, with fewer minimal primes than $R$, and $\bar{R}=\overline{R / I_{1}} \times \overline{R / I_{2}}$. Hence $\bar{R}$ may be computed by computing the integral closure of each $R / I_{i}$.

Case 3. If $R$ is a reduced ring that is not necessarily equidimensional, one may compute the minimal primes $P_{1}, \ldots, P_{n}$ of $R$ using an algorithm for primary decomposition—admittedly an expensive step—and then compute $\bar{R}$ using Case 1 and the fact that $\bar{R}=\overline{R / P_{1}} \times \cdots \times \overline{R / P_{n}}$.

Remark 1.6. Theorem 1.1 may be extended as follows. Suppose a reduced ring $R$ has an endomorphism $\varphi$ with the property that for each valuation $v: R \longrightarrow \mathbb{Z} \cup\{\infty\}$, there exists an integer $k \geqslant 2$ such that

$$
v(\varphi(r))=k v(r) \quad \text { for each } r \in R .
$$

Let $D$ be a nonzerodivisor in the conductor of $R$. Set $V_{0}=\frac{1}{D} R$ and

$$
V_{e+1}=\left\{f \in V_{e} \mid \varphi(f) \in V_{e}\right\} \quad \text { for } e \geqslant 0 .
$$

Imitating the proof of Theorem 1.1, one sees that the descending chain

$$
V_{0} \supseteq V_{1} \supseteq V_{2} \supseteq V_{3} \supseteq \cdots
$$

stabilizes at $\bar{R}$. If colon ideals and kernels of endomorphisms are computable in $R$, then each $V_{e}$ is algorithmically constructible.

As an example, consider a polynomial ring $A=\mathbb{F}\left[x_{1}, \ldots, x_{k}\right]$ over a field $\mathbb{F}$. Let $R$ be a subring of $A$ that is generated, as an $\mathbb{F}$-algebra, by finitely many monomials. Fix an integer $k \geqslant 2$. The $\mathbb{F}$-algebra endomorphism of $A$ with $x_{i} \mapsto x_{i}^{k}$ restricts to an endomorphism $\varphi$ of $R$ satisfying property (1.6.1). Thus, one obtains an algorithm for computing the integral closure of affine semigroup rings; see Bruns and Koch [2001] for another algorithm.

Remark 1.7. The Leonard-Pellikaan algorithm [2003] is based on earlier work of Leonard [2001]. These papers make use of the Frobenius endomorphism along with a weighted total-degree monomial ordering; this is a monomial ordering under which there are only finitely many elements preceding any given element, and this is an essential ingredient in proving the convergence of their algorithm. The affine domains considered in [Leonard and Pellikaan 2003] are constructed as towers in the following sense: $R_{0}$ is a finite field; if $R_{j-1}$ is given with a weight function $\mathrm{wt}_{j-1}$, then $R_{j}$ is the integral closure of $R_{j-1}\left[x_{j}\right] /\left(\varphi_{j}\left(x_{j}\right)\right)$ in $F_{j-1}\left[x_{j}\right] /\left(\varphi_{j}\left(x_{j}\right)\right)$, 
as computed by their algorithm; here $F_{j-1}$ is the field of fractions of $R_{j-1}$, and

$$
\varphi_{j}\left(x_{j}\right)=x_{j}^{m_{j}}+u_{j} \prod_{i=1}^{j-1} x_{i}^{\alpha_{i, j}}+g_{j}\left(x_{j}, \ldots, x_{1}\right)
$$

is an element of $R_{j-1}\left[x_{j}\right]$ that is irreducible and monic in $x_{j}$, such that $u_{j}$ is a nonzero element of $R_{0}$, and the weight function satisfies

$$
\mathrm{wt}_{j}\left(g_{j}\left(x_{j}, \ldots, x_{1}\right)\right)<\mathrm{wt}_{j}\left(x_{j}^{m_{j}}\right)=\mathrm{wt}_{j}\left(\prod_{i=1}^{j-1} x_{i}^{\alpha_{i, j}}\right),
$$

where $\mathrm{wt}_{j}$ is a modification (not a simple extension) of $\mathrm{wt}_{j-1}$ that requires further technical assumptions on the $m_{j}$ and $\alpha_{i, j}$. A complexity analysis of some aspects of the Leonard-Pellikaan algorithm is carried out in [Hu and Maharaj 2008].

\section{Implementation and examples}

Here is our code in Macaulay 2 [Grayson and Stillman], which uses this algorithm to compute the integral closure.

Input: An integral domain $R$ that is finitely generated over a finite field, and, optionally, a nonzero element $D$ of the conductor ideal of $R$.

Output: A set of generators for $\bar{R}$ as a module over $R$.

\section{Macaulay 2 function:}

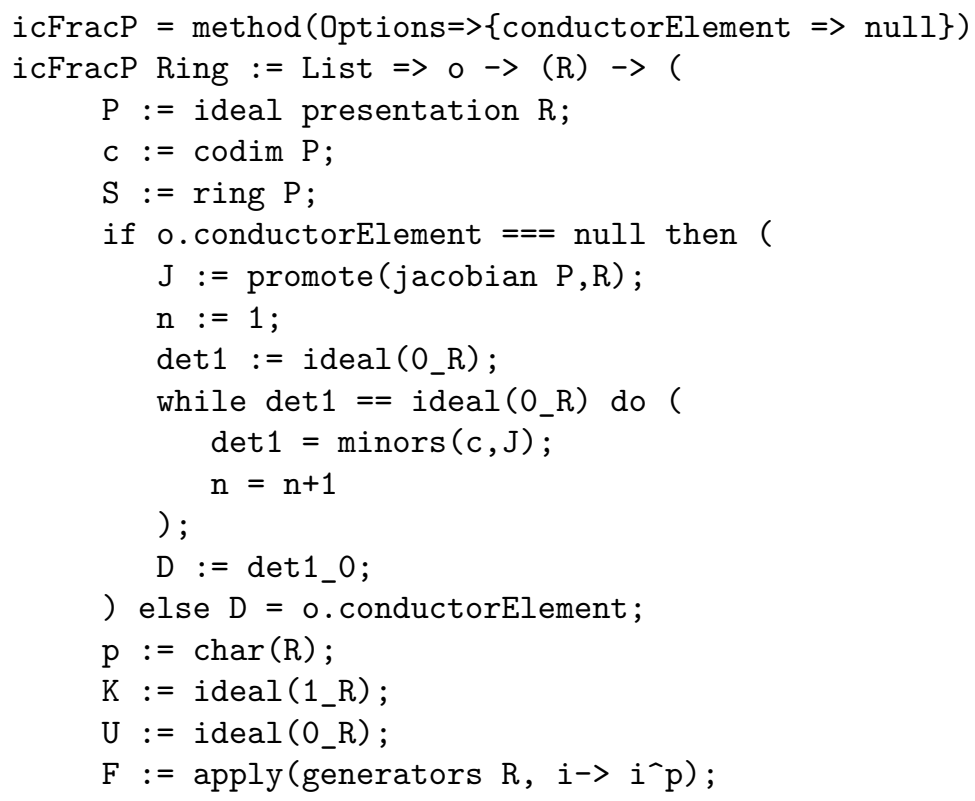




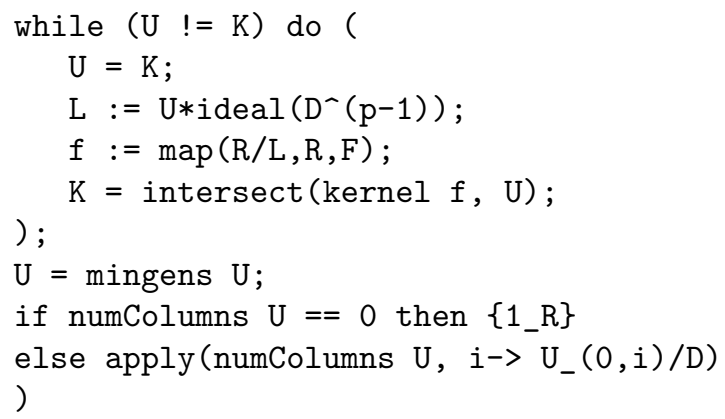

Since the Leonard-Pellikaan algorithm uses the Frobenius endomorphism, it is less efficient when the characteristic of the ring is a large prime. In the examples that follow, the computations are performed on a MacBook Pro computer with a $2 \mathrm{GHz}$ Intel Core Duo processor; the time units are seconds. The comparisons are with de Jong's algorithm [1998] as implemented in the program ICfractions in Macaulay 2, version 1.1.

Example 2.1. Let $\mathbb{F}_{2}[x, y, t]$ be a polynomial ring over the field $\mathbb{F}_{2}$, and set $R=$ $\mathbb{F}_{2}\left[x, y, x^{2} t, y^{2} t\right]$. Then $R$ has a presentation $\mathbb{F}_{2}[x, y, u, v] /\left(x^{2} v-y^{2} u\right)$, which shows, in particular, that $x^{2}$ is an element of the conductor ideal. Setting $D=x^{2}$, the algorithm above computes that the integral closure of $R$ is generated, as an $R$ module, by the elements 1 and $x y t$. Tracing the algorithm, one sees that $V_{0}$ is not equal to $V_{1}$, that $V_{1}$ is not equal to $V_{2}$, and that $V_{2}=V_{3}$. Indeed, these $R$-modules are

$$
V_{0}=\frac{1}{x^{2}} R, \quad V_{1}=\frac{1}{x} R+y t R, \quad V_{e}=R+y t R \text { for } e \geqslant 2 .
$$

As is to be expected, the algorithm is less efficient as the characteristic of the ground field increases:

\begin{tabular}{|lccccccccc|}
\hline characteristic $p$ & 2 & 3 & 5 & 7 & 11 & 13 & 17 & 37 & 97 \\
\hline icFracP & 0.04 & 0.03 & 0.04 & 0.04 & 0.04 & 0.05 & 0.05 & 0.13 & 0.59 \\
icFractions & 0.08 & 0.09 & 0.09 & 0.09 & 0.14 & 0.15 & 0.15 & 0.15 & 0.15 \\
\hline
\end{tabular}

Integral closure of $\mathbb{F}_{p}[x, y, u, v] /\left(x^{2} v-y^{2} u\right)$.

We remark that $R$ is an affine semigroup ring, so its integral closure may also be computed using the program normaliz of Bruns and Koch [2001].

Example 2.2. Consider the hypersurface

$$
R=\mathbb{F}_{p}[u, v, x, y, z] /\left(u^{2} x^{4}+u v y^{4}+v^{2} z^{4}\right) .
$$

It is readily verified that $R$ is a domain, and that $t=u x^{4} / v$ is integral over $R$. The ring $R[t]$ has a presentation $\mathbb{F}_{p}[u, v, x, y, z, t] / I$, where $I$ is the ideal generated 
by the $2 \times 2$ minors of the matrix

$$
\left(\begin{array}{ccc}
u & t & -z^{4} \\
v & x^{4} & t+y^{4}
\end{array}\right)
$$

Since the entries of the matrix form a regular sequence in $\mathbb{F}_{p}[u, v, x, y, z, t]$, the ring $R[t]$ is Cohen-Macaulay. Moreover, if $p \neq 2$, then the singular locus of $R[t]$ is $V(t, y, x z, v z, u x)$ which has codimension 2 , so $R[t]$ is normal.

If $p=2$ then the ring $R[t]$ is not normal; indeed, in this case, the integral closure of $R$ is generated, as an $R$-module, by the elements

$$
1, \quad \sqrt{u v}, \quad \frac{u x+z \sqrt{u v}}{y}, \quad \frac{v z+x \sqrt{u v}}{y}, \quad \frac{u x z+z^{2} \sqrt{u v}}{u y} .
$$

For small values of $p$, these computations may be verified on Macaulay 2 using either algorithm; some computations times are recorded next. (Here and in the next table $*$ means that the computation did not terminate within six hours.)

\begin{tabular}{|lccccc|}
\hline characteristic $p$ & 2 & 3 & 5 & 7 & 11 \\
\hline icFracP & 0.07 & 0.22 & 9.67 & 143 & 12543 \\
icFractions & 1.16 & $*$ & $*$ & $*$ & $*$ \\
\hline
\end{tabular}

Integral closure of $\mathbb{F}_{p}[u, v, x, y, z] /\left(u^{2} x^{4}+u v y^{4}+v^{2} z^{4}\right)$.

Example 2.3. Consider the hypersurface

$$
R=\mathbb{F}_{p}[u, v, x, y, z] /\left(u^{2} x^{p}+2 u v y^{p}+v^{2} z^{p}\right),
$$

where $p$ is an odd prime. We shall see that $\bar{R}$ has $p+1$ generators as an $R$-module, but first some comparisons:

\begin{tabular}{|lcccccccc|}
\hline characteristic $p$ & 3 & 5 & 7 & 11 & 13 & 17 & 19 & 23 \\
\hline icFracP & 0.07 & 0.09 & 0.27 & 1.81 & 4.89 & 26 & 56 & 225 \\
icFractions & 1.49 & 75.00 & 4009 & $*$ & $*$ & $*$ & $*$ & $*$ \\
\hline
\end{tabular}

Integral closure of $\mathbb{F}_{p}[u, v, x, y, z] /\left(u^{2} x^{p}+2 u v y^{p}+v^{2} z^{p}\right)$.

We claim that $\bar{R}$ is generated, as an $R$-module, by the elements

$$
1, \sqrt{y^{2}-x z} \text {, and } u^{i / p} v^{(p-i) / p} \text { for } 1 \leqslant i \leqslant p-1 .
$$

It is immediate that these elements are integral over $R$; to see that they belong to the fraction field of $R$, note that

$$
\sqrt{y^{2}-x z}= \pm \frac{u y^{p}+v z^{p}}{u\left(y^{2}-x z\right)^{(p-1) / 2}}
$$


and that, by the quadratic formula, one also has

$$
\left(\frac{u}{v}\right)^{1 / p}=\frac{-y \pm \sqrt{y^{2}-x z}}{x} .
$$

Moreover, using (2.3.2), it follows that

$$
v^{1 / p} \sqrt{y^{2}-x z}= \pm\left(x u^{1 / p}+y v^{1 / p}\right),
$$

and hence the $R$-module generated by the elements (2.3.1) is indeed an $R$-algebra. It remains to verify that the ring

$$
A=R\left[\sqrt{y^{2}-x z}, u^{i / p} v^{(p-i) / p} \mid 1 \leqslant i \leqslant p-1\right]
$$

is normal. For this, it suffices to verify that

$$
B=R\left[\sqrt{y^{2}-x z}, u^{1 / p}, v^{1 / p}\right]
$$

is normal, since $A$ is a direct summand of $B$ as an $A$-module: use the grading on $B$ where $\operatorname{deg} x=\operatorname{deg} y=\operatorname{deg} z=0$ and $\operatorname{deg} u^{1 / p}=1=\operatorname{deg} v^{1 / p}$, in which case $A$ is the $p$ th Veronese subring $\bigoplus_{i \in \mathbb{N}} B_{i p}$. The ring $B$ has a presentation $\mathbb{F}_{p}[x, y, z, d, s, t] / I$, where $I$ is generated by the $2 \times 2$ minors of the matrix

$$
\left(\begin{array}{ccc}
y+d & z & s \\
x & y-d & -t
\end{array}\right)
$$

and $s \mapsto u^{1 / p}, t \mapsto v^{1 / p}, d \mapsto \sqrt{y^{2}-x z}$. But then - after a change of variables $B$ is a determinantal ring, and hence normal.

\section{Acknowledgment}

We are very grateful to Douglas Leonard for drawing our attention to [Leonard and Pellikaan 2003] and answering several questions, to David Eisenbud, Ruud Pellikaan, and Wolmer Vasconcelos for their feedback, and to Amelia Taylor for valuable discussions and help with Macaulay 2.

\section{References}

[Bruns and Koch 2001] W. Bruns and R. Koch, "Computing the integral closure of an affine semigroup”, Univ. Iagel. Acta Math. 39 (2001), 59-70. MR 2002m:20095 Zbl 1006.20045

[Grayson and Stillman] D. R. Grayson and M. E. Stillman, Macaulay 2, a software system for research in algebraic geometry, Available at http://www.math.uiuc.edu/Macaulay2.

[Hu and Maharaj 2008] X. Hu and H. Maharaj, "On the $q$ th power algorithm", Finite Fields Appl. 14:4 (2008), 1068-1082. MR 2009g:14028 Zbl 1153.14022

[Huneke and Swanson 2006] C. Huneke and I. Swanson, Integral closure of ideals, rings, and modules, London Mathematical Society Lecture Note Series 336, Cambridge University Press, 2006. MR 2008m:13013 Zbl 1117.13001 
[de Jong 1998] T. de Jong, "An algorithm for computing the integral closure", J. Symbolic Comput. 26:3 (1998), 273-277. MR 99d:13007 Zbl 0932.13021

[Leonard 2001] D. A. Leonard, "Finding the defining functions for one-point algebraic-geometry codes", IEEE Trans. Inform. Theory 47:6 (2001), 2566-2573. MR 2003d:94122 Zbl 1019.94030

[Leonard and Pellikaan 2003] D. A. Leonard and R. Pellikaan, "Integral closures and weight functions over finite fields”, Finite Fields Appl. 9:4 (2003), 479-504. MR 2005d:13015 Zbl 1085.11059

[Lipman and Sathaye 1981] J. Lipman and A. Sathaye, "Jacobian ideals and a theorem of BriançonSkoda”, Michigan Math. J. 28:2 (1981), 199-222. MR 83m:13001 Zbl 0438.13019

[Seidenberg 1970] A. Seidenberg, "Construction of the integral closure of a finite integral domain", Rend. Sem. Mat. Fis. Milano 40 (1970), 100-120. MR 45 \#3396 Zbl 0218.14023

[Seidenberg 1975] A. Seidenberg, "Construction of the integral closure of a finite integral domain. II”, Proc. Amer. Math. Soc. 52 (1975), 368-372. MR 54 \#12741 Zbl 0333.13004

[Stichtenoth 1993] H. Stichtenoth, Algebraic function fields and codes, Springer, Berlin, 1993. MR 94k:14016 Zbl 0816.14011

[Stolzenberg 1968] G. Stolzenberg, "Constructive normalization of an algebraic variety", Bull. Amer. Math. Soc. 74 (1968), 595-599. MR 37 \#201 Zbl 0164.04202

[Vasconcelos 1991] W. V. Vasconcelos, "Computing the integral closure of an affine domain", Proc. Amer. Math. Soc. 113:3 (1991), 633-638. MR 92b:13013 Zbl 0739.13014

[Vasconcelos 2000] W. V. Vasconcelos, "Divisorial extensions and the computation of integral closures”, J. Symbolic Comput. 30:5 (2000), 595-604. MR 2001k:13017 Zbl 0999.13004

Communicated by Kei-Ichi Watanabe

Received 2008-11-13 Revised 2009-05-11 Accepted 2009-06-11

singh@math.utah.edu

University of Utah, Department of Mathematics, 155 South 1400 East, Salt Lake City, UT 84112-0090, United States http://www. math.utah.edu/ singh/

iswanson@reed.edu

Reed College, Department of Mathematics, 3203 SE Woodstock Boulevard, Portland, OR 97202-8199, United States http://people.reed.edu/ iswanson/ 